A comparison between maximum likelihood and Bayesian estimation of stochastic frontier production models

FCO. JAVIER ORTEGA IRIZO

e-mail: fjortega@us.es

Phone: +34954556970

JOSÉ MANUEL GAVILÁN RUIZ

e-mail: gavi@us.es

Phone: +34954556970

Address (for both authors):

Departamento de Economía Aplicada I. UNIVERSIDAD DE SEVILLA

Avenida Ramón y Cajal, nº1. 41018-Sevilla.

Author for correspondence: José Manuel Gavilán Ruiz. 


\title{
A comparison between maximum likelihood and Bayesian estimation of stochastic frontier production models
}

\begin{abstract}
In this paper, the finite sample properties of the maximum likelihood and Bayesian estimators of the half-normal stochastic frontier production function are analysed and compared, through a Monte Carlo study. The results show that the Bayesian estimator should be used in preference to the maximum likelihood owing to the fact that the mean square error performance is substantially better in the Bayesian framework.
\end{abstract}

Key words: stochastic frontier, Monte Carlo, maximum likelihood, Bayesian estimator

AMS Classification: 62-04

JEL Classification: C11, C15, D24. 


\section{INTRODUCTION}

The origin of the analysis of frontier production models in econometrics and the calculation of efficiency measures can be set in the pioneering work of Farrell (1957), where the original idea of analysing the efficiency of a productive process in terms of the observed deviations between actual production and an ideal output frontier was established. In econometric terms, such deviations can be identified with the random perturbations of a regression model.

Following on from Farrell's idea, Aigner and Chu (1968) propose a model where a certain output is expressed as a function of several inputs and unknown parameters together with a negative random perturbation; the deterministic part of the model represents the production frontier or maximum attainable output value for a given level of inputs, while the random perturbation (the difference between the actual production and the maximum attainable) is the degree of inefficiency of the productive process.

This framework, usually called the deterministic frontier production model, presents some difficulties due to the fact that the unilateral perturbation fails to verify the standard hypothesis of regularity needed to obtain the asymptotic properties of the maximum likelihood estimator (ML) (Ortega and Basulto, 2009; Ortega et al. 2009). Furthermore, this modelling takes no account of possible sources of error measures and other kinds of random variations due to the inefficiency of the production process.

In order to provide an answer to latter problem, Aigner et al. (1977), Battese and Corra (1977), and Meusen and Van der Broeck (1977) formulated econometric models with a composite error term; currently known as stochastic frontier production models. In this framework, two random perturbations are considered; one is a measure of inefficiency, and the other represents all the possible sources of random variations.

In these kinds of models, the standard regularity conditions are verified. As a consequence, the asymptotic properties of the ML estimator can be used to carry out inferences. Moreover, the specific software developed, (such as FRONTIER and LIMPDEP), has propagated the use of this type of models. 
As an alternative, the Bayesian approach can be used to estimate the parameters of the model and the inefficiency (or efficiency) of each firm considered. Bayesian methodology presents some advantages, as for example its simplicity in incorporating parameter constraints. Above all, the use of Monte Carlo methods and Markov Chains together with Gibbs' algorithm (Gelfand and Smith, 1990) provides a very suitable tool in this framework due to the complexity of stochastic frontier models. In fact, a major part of recent literature uses the Bayesian approach (Griffin and Steel 2007, Dorfman and Koop 2005).

One of the main drawbacks of the Bayesian approach, especially for practical purposes, is the lack of easy-to-use software which is sufficiently versatile to accommodate the different situations and sets of data. In Griffin and Steel (2007), free software, WinBUGS, is used to estimate the numerous variations of stochastic frontier production models. It is proved that this software is a powerful and flexible tool in this context, can be used easily by the practitioners, and that it estimates the models in a reasonable time. Obviously, a specific implementation of a particular model could be more efficient than WinBUGS, since this is a generic tool that can be used in a large variety of situations. However, the ease of use and the possibility of performing a Bayesian analysis without programming are clear advantages.

The main goal of this article is to carry out a simulation analysis to compare the results of the ML method (using the software FRONTIER) with those obtained through the Bayesian approach (using the software WinBUGS). To this end, since either method can be integrated into the free software $\mathrm{R}$, the comparison of the results obtained with either of the two methods is more reliable, since they can be applied to the same simulated samples. Our design of the Monte Carlo experiment is similar to that of Coelli (1995), where the ML and corrected least-squares estimators are compared. Zhang (1999) also compares Bayesian and ML estimation, although this is performed by fixing the values of the parametric space and by placing the stress on analysing the effect of using different prior distributions. In Ortega et al. (2009) a comparison between the two methods is carried out on a deterministic frontier model. 
Although the bias of the estimators is also analysed in this paper, the mean squared error (MSE) is taken as the main comparison criterion, laying special emphasis on the parameter that represents the proportion of variance of the composite error that is due to inefficiency, and on the estimation of the individual inefficiencies (or efficiencies).

From here on, the article is organised as follows: In Section 2 the production model is present, upon which the analysis is performed, the likelihood function under the assumed hypothesis for the perturbation term, and the parameterisation used to carry out the estimation process; In Section 3, a way to obtain the ML and Bayesian estimators using FRONTIER and WinBUGS respectively is explained, always in the setting of the software R. Section 4 is devoted to describing the design of the Monte Carlo experiment. In Section 5 , the results obtained and an interpretation of the most interesting points are presented. Finally, the main conclusions of this work are laid out in Section 6.

\section{THE MODEL}

The basic formulation of the stochastic frontier production model is:

$$
y_{i}=f\left(x_{i}, \beta\right)+\underbrace{v_{i}-u_{i}}_{\varepsilon_{i}}, \quad i=1, \ldots, n,
$$

where $y_{i}$ is the production of the $i$-th firm, $x_{i}$ is the vector of all its inputs, $\beta$ is a vector of parameters to be estimated, and $f(\bullet)$ is the production function.

The random perturbation $\varepsilon_{i}$ consists of two parts (composite error), $v_{i} \in \mathbb{R}$, which represents the sources of random variations and $u_{i}>0$, which is the inefficiency of the production process. It is commonly supposed that $v_{i}$ follows a Normal distribution, specifically $v_{i} \sim N\left(0, \sigma_{v}^{2}\right)$, although with regard to $u_{i}$, a positive probability distribution must be chosen. In this work, since the most usual hypothesis is supposed, that is $u_{i}=\left|u_{i}^{*}\right|$, where $u_{i}^{*} \sim N\left(0, \sigma_{u}^{2}\right)$, then it is said that the perturbations $u_{i}$ follow a Half-Normal distribution and is written $u_{i} \sim H N\left(0, \sigma_{u}^{2}\right)$. Moreover, it is supposed that all perturbations ( $v_{i}$ and $u_{i}$ ) are independent. Other kinds of probability densities for the perturbations $u_{i}$, such as Exponential and Gamma distributions, have been frequently and widely used, 
mainly when the Bayesian approach is considered, (Koop et al. 1995, Osiewalski and Steel 1998, Koop and Stell 2003).

With regard to the production function, the most usual situation is considered: $f\left(x_{i}, \beta\right)=x_{i}^{\prime} \beta$, where $\beta$ is an unknown vector of parameters with an intercept. It should be observed that if $x_{i}$ and $y_{i}$ are measured on a logarithmic scale, then a Cobb-Douglas production function is obtained. In this case, the most common one, instead of being interested on $u_{i}$ as an inefficiency measure, $\exp \left(-u_{i}\right)$ is usually estimated, which is an efficiency measure bounded between 0 and 1 .

In order to obtain the ML estimator, the parameterization $\sigma^{2}=\sigma_{v}^{2}+\sigma_{u}^{2}$ and $\gamma=\sigma_{u}^{2} / \sigma^{2}$ is considered in the frontier package. Hence, the parameter $\gamma$ takes values between 0 and 1 and shows the proportion of the variance due to inefficiency. In fact, $\gamma$ is not exactly such a proportion of variance, since $\operatorname{var}[u]=p \sigma_{u}^{2}$ with $p=(\pi-2) / \pi$. To be more precise, if $\gamma^{*}$ denotes the proportion of the total variance owing to the inefficiency, (that is $\gamma^{*}=\operatorname{var}[u] /(\operatorname{var}[u]+\operatorname{var}[v])$ ), then it is straightforward to verify that $\gamma^{*}=\gamma /\left(\gamma+(1-\gamma) p^{-1}\right)$.

With this parameterization, the logarithm of the likelihood for the i-th observation is given by:

$$
\log \left(L_{i}\right)=-\frac{1}{2} \log \left(\frac{\pi}{2}\right)-\log (\sigma)-\frac{1}{2} z_{i}^{2}+\log \left(\Phi\left(-z_{i} \sqrt{\gamma /(1-\gamma)}\right)\right)
$$

where $z_{i}=\frac{y_{i}-x_{i}^{\prime} \beta}{\sigma}$

\section{PROGRAMMING THE ESTIMATION METHODS.}


As pointed out before, ML estimation is carried out by using FRONTIER software and Bayesian estimation through the program WinBUGS, both integrated in the environment of the software R.

To be more specific, to obtain the ML estimator, the version 0.996-6 of the frontier package, which uses the FORTRAN source code of the software FRONTIER 4.1 (Coelli, 1996), is used. For the Bayesian inference, the package R2WinBUGS is employed, which opens the WinBUGS v1.4 program, passes it the data, picks up the results and delivers them to the R environment. The WinBUGS software can be downloaded from the Web site http://www.mrc-bsu.cam.ac.uk/bugs/winbugs/contents.shtml and the R software can be obtained from http://www.r-project.org, both at no cost. After installing R, the packages frontier and $R 2$ WinBUGS must be installed and loaded. For details and options of these packages, the help included within them can be consulted. A detailed explanation of how the package $R 2$ WinBUGS works, together with some examples, can be found in Sturtz et al. (2005).

For the sake of simplicity, a single explanatory variable plus an intercept are considered in the design of the simulation experiment. That is to say, the simulations are made on the basis of the model:

$$
y_{i}=\beta_{0}+\beta_{1} x_{i}+v_{i}-u_{i}, \quad i=1, \ldots, n .
$$

The actual instructions used in $\mathrm{R}$ together with a short explanation are presented in Appendix 1A.

In order to obtain the Bayesian estimations (of the parameters and of the efficiencies), the prior distributions used in Griffin and Steel (2007) are considered, except for the hyperparameter for the variance of the Half-Normal distribution. Specifically, for the parameters $\beta_{0}, \beta_{1}$, and $\sigma_{v}^{-2}$, the non-informative distributions $\beta_{0} \sim N\left(0,10^{6}\right)$, $\beta_{1} \sim N\left(0,10^{6}\right)$ and $\sigma_{v}^{-2} \sim G a\left(10^{-3}, 10^{-3}\right)$ are taken, with $N\left(\mu, \delta^{2}\right)$ representing a Normal distribution with mean $\mu$ and variance $\delta^{2}$, and where $G a\left(a_{0}, a_{1}\right)$ is a Gamma distribution with mean $a_{0} / a_{1}$, and shape parameter $a_{0}$. 
The prior distribution of the parameter $\sigma_{u}^{-2}$ is commonly chosen as that belonging to the Gamma family, setting the hyperparameters such that the prior median takes into consideration our beliefs about the median efficiency of the production process under analysis (Van den Broeck et al. 1994, Zhang, X. 1999). Griffin and Steel (2007), in their example, which was applied to the USA electricity market, take the prior distribution $\sigma_{u}^{-2} \sim G a(1,0.0267)$, which implies median efficiency in the sector of 0.875 is assumed. In this work, taking into account that a simulation process is carried out, a benchmark value of 0.5 for the prior median efficiency is chosen, which is achieved with a distribution $\sigma_{u}^{-2} \sim G a(1,0.7216)$ (Van de Broeck et al. 1994).

With regard to the implementation of the model in WinBUGS, three Markov chains of 1500 iterations each are generated, the first 500 of each of which are dismissed; this implies a total of 3000 simulated samples. As starting points, the Corrected Least-Squares estimators are taken for the parameters $\beta_{0}$ and $\beta_{1}$ (Green, 1980). For the rest of the parameters, random values are generated.

The text file with the necessary instructions for the definition of the model in WingBUGS, and the code implemented in R to carry out the Bayesian estimation, are given in Appendix 1B.

\section{DESIGN OF THE MONTE CARLO EXPERIMENT.}

As stated in the former Section, the simulations are made on a model with one explanatory variable plus an intercept. The sample space of the experiment is initially given by the different values of the parameters $\beta_{0}, \beta_{l}, \sigma^{2}$ and $\gamma$, the sample size $n$ and the values of the vector of observations of the covariable $x$. The main goal is to analyse the behaviour of the estimations of both the parameter $\gamma$ and of the individual efficiencies.

Without any loss of generality, the values of $\beta_{0}, \beta_{1}$ and $\sigma^{2}$ can be chosen (Zhang 1999, Coelli, 1995). Hence, in this work $\beta_{0}=\beta_{1}=\sigma^{2}=1$ is selected. With respect to the values of the covariable $x$, these are generated uniformly on the interval $[0,10]$. Since the intention is to analyse the behaviour of both methods in small and large sample sizes, 
$n \in\{10,20,50,100,500\}$ is taken. With regard to the parameter $\gamma$, following Coelli (1995), the values of the parameter $\gamma^{*}$ are set instead. Specifically values ranging from 0 to 1 with increments of 0.1 have been chosen. From the equation $\gamma^{*}=\gamma /\left(\gamma+(1-\gamma) p^{-1}\right)$ the following relation between $\gamma$ and $\gamma^{*}$ is obtained:

\begin{tabular}{c|c|c|c|c|c|c|c|c|c|c|c}
$\gamma^{*}$ & 0.0 & 0.1 & 0.2 & 0.3 & 0.4 & 0.5 & 0.6 & 0.7 & 0.8 & 0.9 & 1.0 \\
\hline$\gamma$ & 0.00 & 0.24 & 0.41 & 0.54 & 0.65 & 0.73 & 0.80 & 0.87 & 0.92 & 0.96 & 1.00
\end{tabular}

As a consequence, a total of 11 values of $\gamma$ and 5 values of $n$ are simulated, which yields 55 combinations. For each one of such combinations, a total of $m=1000$ replications of the model are carried out. In order to obtain pseudorandom numbers, the default generator implemented in the software R is used. The ML and Bayesian estimators are calculated as explained in the section above.

For each parameter, the mean bias (MB) and the mean squared error (MSE) of each of the $m$ replications with each of the methods are obtained. In order to analyse the efficiencies, the study is not limited to estimating only the mean efficiency but the MB and MSE of each individual efficiency are also calculated. Therefore, as an overall indicator, the average of the MB and the MSE for each firm are given, that is, if $E_{i}$ is the efficiency of the i-th firm and $\hat{E}_{i j}$ its corresponding estimated value, (by either of the methods), in the j-th replication, then $M B_{i}=m^{-1} \sum_{j=1}^{m}\left(\hat{E}_{i j}-E_{i}\right)$ and $M S E_{i}=m^{-1} \sum_{j=1}^{m}\left(\hat{E}_{i j}-E_{i}\right)^{2}$, for $i=1, \ldots, n$. Hence, the $\mathrm{MB}$ and the MSE of the efficiencies are obtained by $M B=n^{-1} \sum_{i=1}^{n} S M_{i}$ and $M S E=n^{-1} \sum_{i=1}^{n} E C M_{i}$.

Before analysing the results, the main differences between our Monte Carlo experiment and that carried out by Zhang (1999) should be pointed out. Firstly, this author uses a specific algorithm for the Bayesian estimation, whereas in this work, general but very easy-to-use Bayesian software is employed, which makes it more appealing for practitioners (even though more processing time is possibly required to obtain the estimates in each replication). Secondly, in Zhang (1999) only one single value of the parametric space is considered, and the main interest is the analysis of the influence of the choice of various hyperparameters in the prior distribution of $\sigma_{u}^{2}$ exerted on the bias and 
on the mean squared error of the parameters of the model. However, in this work only one prior distribution is considered for all cases and the behaviour of the two methods for various values of parameter $\gamma$ is analysed, with special attention paid to the individual efficiencies estimated.

\section{RESULTS OF THE MONTE CARLO EXPERIMENT.}

The complete results of the Monte Carlo experiment, that is to say, the bias and the MSE of all the parameters and the individual efficiencies in the 55 cases considered, are offered in Appendix 2. Here, the most relevant results are presented, while attention is focused on the MSE criterion, which indicates which methodology better replicates the true values of the considered model. The behaviour of the estimations of parameter $\gamma$ and the individual efficiencies are our main concern. As already pointed out, parameter $\gamma$ captures the structure of the composite error. It is common knowledge that one of the main drawbacks of this kind of model is the difficulty in identifying what proportion of the total error is due to inefficiency and what proportion is due to random effects. Moreover, the correct estimation of $\gamma$ is crucial for the attainment of the individual efficiencies of each firm, which constitutes one of the main objectives when using this kind of model.

In Figure 1, the MSE of parameter $\gamma$, in accordance with the values of $\gamma^{*}$, is shown for various sample sizes and for the two methods of estimation, (the case $n=10$ has been omitted in order to prevent complications in the interpretation for the rest of the sample sizes, since otherwise the ordinate axis would have to be increased). With respect to the ML method, the graph is in accordance with Figure 2 in Coelli (1995). In fact, the results are virtually coincident for the sizes $n=50$ and $n=100$, while for $n=500$, it is very similar to that presented by Coelli (1995) for $n=400$. 

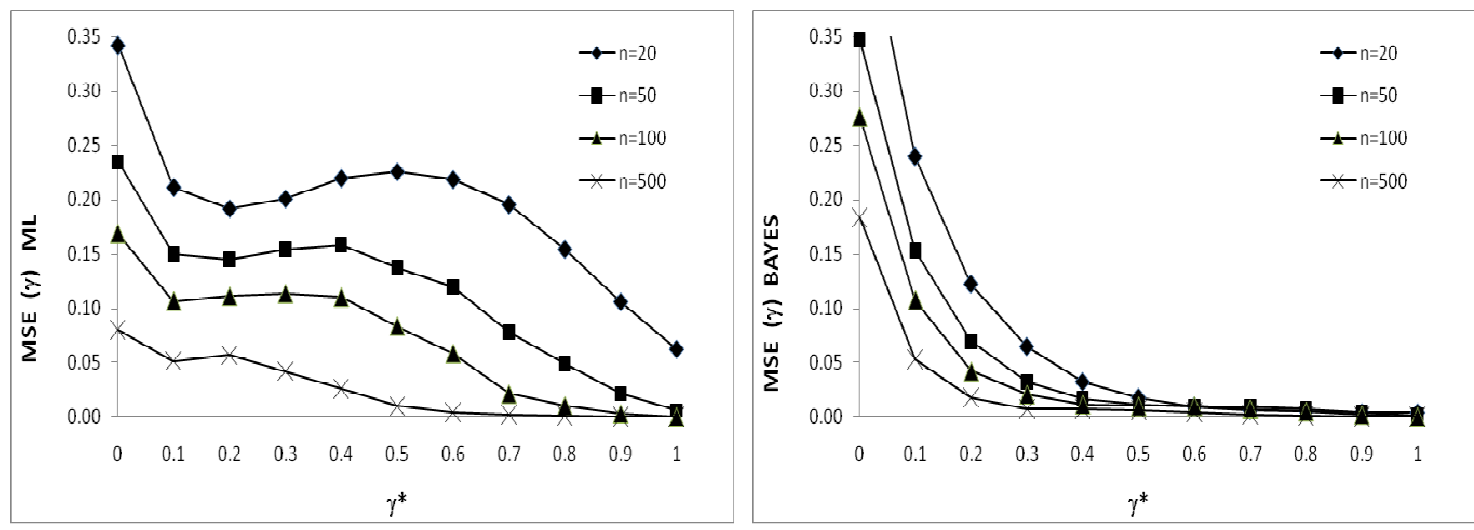

Fig. 1: MSE of parameter $\gamma$ for the two estimation methods.

As can be clearly seen, the behaviour of the MSE in the Bayesian methodology is totally different from that of the ML estimator; the former being better than the latter. Indeed, although for the extreme case $\gamma^{*}=0$, the Bayesian MSE is greater than the ML MSE, from $\gamma^{*}=0.2$ the Bayesian MSE quickly falls, and is lower than that for the respective ML except for the values $\gamma^{*}=0.9$ and $\gamma^{*}=1$ in which the MSE tend to be equal (in the case $n=500$, the values of MSE tend to be equal from $\gamma^{*}=0.5$ ). Therefore, the Bayesian approach shows better behaviour in most of the parametric space of $\gamma^{*}$. It is also interesting to observe that the MSE in the Bayesian case always decreases as the values of $\gamma^{*}$ increase, which is not always true for the ML estimator.

Let us also observe that, as expected, the MSE of the two estimation methods decreases with the sample size. This behaviour is shown by all the parameters analysed.

In Figure 2, the results are given of a similar analysis but this time as applied to the individual efficiencies. In all cases, the MSE of the individual efficiencies is first obtained, and their overall mean is calculated. This average is given in the results and depicted in Figure 2. The observations made for Figure 1 remain valid for Figure 2, since the pattern of behaviour displayed is the same, although in the case of the efficiencies, the scale of the MSE is smaller in the two approaches. 

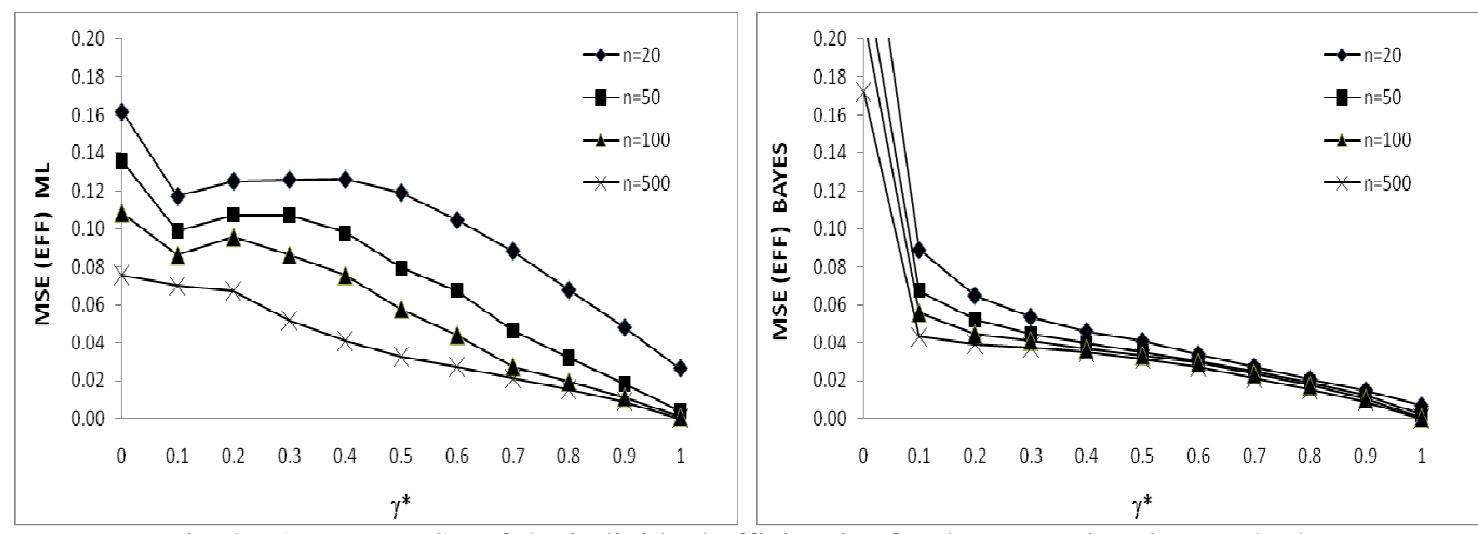

Fig. 2: Average MSE of the individual efficiencies for the two estimation methods.

The similarity between Figures 1 and 2 reveals the direct influence that the estimation of the parameter $\gamma$ has on the estimation of the efficiencies of the firms.

In order to quantify the difference in the behaviour of the two estimation methods, in Figure 3 the ratio between the MSE of maximum likelihood and the Bayesian MSE for $\gamma$ and for the efficiencies have been depicted. Values of these ratios above 1 are identified with a better behaviour of the Bayesian estimator, while on the other hand, a value below 1 indicates a better performance of the ML estimator. The sample size $n=20$ has been omitted from the graph in order to maintain the scale within the axis of ordinates.
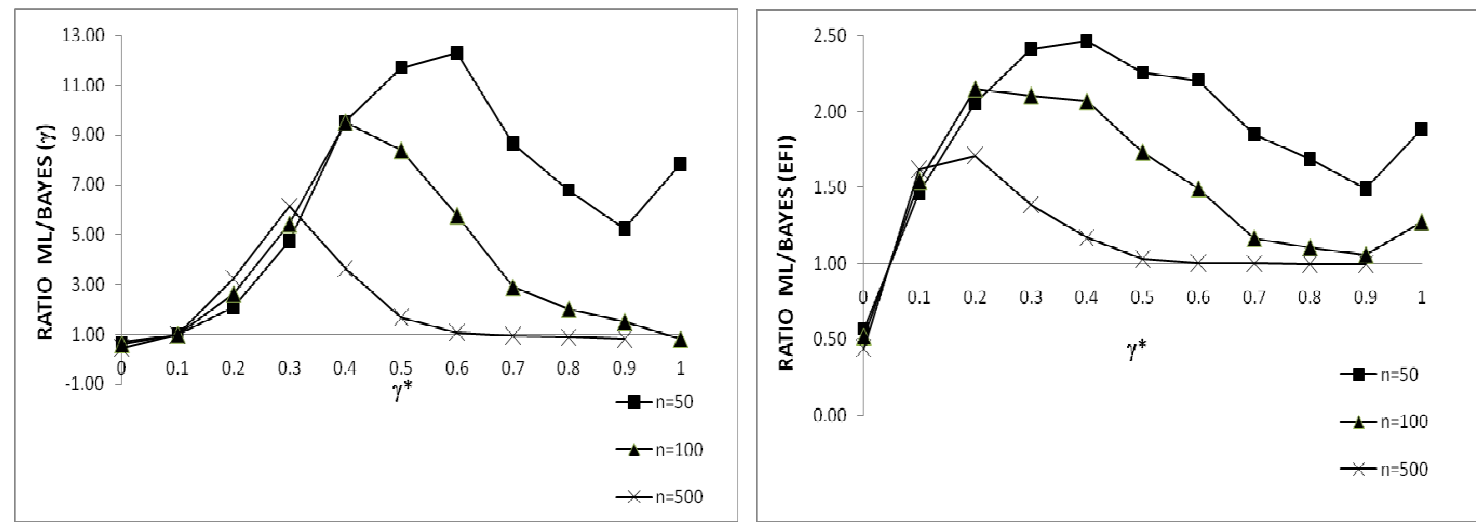

Fig. 3: Ratios of the MSE for $\gamma$ and for the efficiencies.

As shown in Figures 1 and 2, the behaviour of the Bayesian estimator is consistently better except for the extreme case $\gamma^{*}=0$. Moreover, Figure 3 reveals that the differences in the results for the two methods are highly significant. For instance, for the parameter $\gamma$ and with a sample size $n=50$, around the value $\gamma^{*}=0.6$ the MSE of the ML estimator is 
the order of 12 times greater than that of the Bayesian counterpart. Such differences remain significant for the case $n=100$ and even when $n=500$, since ratios of the order from 3 to 6 are observed when $\gamma^{*}$ ranges between 0.2 and 0.4. With regard to the efficiencies, a similar behaviour to that of parameter $\gamma$ is shown, although on a much smaller scale. In this case, the greatest observed ratios are in the region of 2.5 , all of which (except $\gamma^{*}=0$ ) remain favourable to the Bayesian estimator.

An indication of the significance of the differences in the MSE's is provided if it is assumed that these MSE ratios have approximate F-distributions (Coelli, 1995), and hence, of the 55 ratios considered for parameter $\gamma, 33$ indicate that the MSE of the Bayesian estimator is significantly smaller than the MSE of ML (at the $1 \%$ level), while in only one case $\left(\gamma^{*}=0\right.$ and $\left.n=500\right)$ do we observe the MSE of ML to be significantly smaller than the Bayesian MSE.

It is worth bearing in mind that for $\gamma^{*}=0.1$, the ratio corresponding to $\gamma$ is nearly 1 for all sample sizes, while the MSE ratio of the efficiencies is around 1.5 for all the values of $n$. It is also important to mention that in the graph, the ratios for $\gamma^{*}=1$ and $n=500$ have been omitted, since the values of the MSE are both so close to 0 that their quotients are meaningless (specifically, the ratios obtained in this case are 12.3 for the parameter $\gamma$ and 3.08 for the efficiency).

According to the comparison criterion used, the results show a clear difference in favour of the Bayesian methodology, which is why we recommend its practical use for the estimation of the stochastic frontier production model considered in this work.

In order to complete the analysis, certain general observations about the MSE and MB on the other parameters should be mentioned.

With regard to the slope, $\beta_{1}$, the main conclusion is that both methods estimate it correctly, since the biases and the MSE are nearly 0 in all cases. With respect to the intercept, $\beta_{0}$, this exhibits a smaller MSE for the Bayesian methodology except for the case $\gamma^{*}=0$. The MSE of the parameter $\sigma^{2}$ displays a more diverse behaviour, since for the sample sizes $n=10$ and $n=20$, it almost always remains lower for the ML estimator, 
whereas for $n=50, n=100$ and $n=500$, the Bayesian MSE is lower than the ML estimator from $\gamma^{*}=0.3$ upwards.

With respect to the behaviour of the biases (considered in absolute values), the most noteworthy conclusion is that the differences between the two methods depend to a greater extent on the values of $\gamma^{*}$ (except for the case of the slope $\beta_{1}$ in which the biases are always similar). For the parameters $\beta_{0}$ and $\gamma$, and for the efficiency, the bias of the ML estimator tends to be smaller when $\gamma^{*} \leq 0.4$ and the opposite is obtained from $\gamma^{*}=0.5$ upwards. However, the ML bias of the parameter $\sigma^{2}$ is in general lower than the Bayesian bias for $\gamma^{*} \leq 0.8$. Therefore, according to the absolute value of the bias criterion, no method is clearly better than the other. As for the sign of the biases, the most outstanding conclusion is that in general the same pattern is followed by both methods, although the change of the sign does not occur in the same values of $\gamma^{*}$. For example, the biases of the parameter $\gamma$ begin as positive for both methods for the first values of $\gamma^{*}$, and they all end up as negative; however the change in the sign for the ML estimator occurs in the region of $\gamma^{*}=0.2$ whereas in the Bayesian case this occurs in the region of $\gamma^{*}=0.6$.

\section{CONCLUSIONS.}

The main conclusion of this work is that the Bayesian estimation is in general preferable to the ML estimation in the analysed stochastic frontier production model. This conclusion has been reached on the basis that the MSE of the parameter $\gamma$ and the estimated efficiencies are lower in the Bayesian approach except for the extreme case in which the sample comes from a model where the whole error is due to random effects (that is, in the case of a lack of inefficiency).

It is also important to point out that the Bayesian estimation has been carried out using the general purpose Bayesian software WinBUGS, utilised inside the R environment, which make its use feasible and appealing to practitioners with only a basic knowledge of the Bayesian statistics. It is with this purpose in mind that the code necessary for the estimation of the model is presented in Appendix 1. 
The results of the simulation have been obtained using the same prior distribution in all cases; for the parameters $\beta_{0}, \beta_{1}$ and $\sigma_{v}^{-2}$ non-informative distributions have been utilised and for the parameter $\sigma_{u}^{-2}$, a distribution has been chosen that assumes a prior median efficiency equal to 0.5. When developing specific applications, researchers can include their previous knowledge about the sector, for example adjusting the prior distribution of $\sigma_{u}^{-2}$ in order to adapt it to their beliefs about the median efficiency. This additional information may well improve the accuracy of the Bayesian estimation. In the case in which the researcher does not have this information, it has been proved with the distribution used, that the Bayesian methodology displays a better behaviour than that for the ML methodology in practically the whole parametric space.

To conclude, let us observe that the analysis has been focused on a model with crosssectional data and perturbations $u_{i}$ following a Half-Normal model. Therefore, natural extensions of this work would be obtained by supposing other kinds of models for the perturbations $u_{i}$, and by considering panel data. 


\section{APPENDIX 1.}

In this appendix, the code used in R to obtain the ML and Bayes estimations of the model $y_{i}=\beta_{0}+\beta_{1} x_{i}+v_{i}-u_{i}, \quad i=1, \ldots, n$ is presented. The packages frontier and R2WinBUGS must first be installed and loaded.

\section{A) ML estimation.}

Firstly, two vectors $x$ and $y$ containing the data are created. In order to obtain the estimations of the parameters of the stochastic frontier production model and its individual efficiencies through the LM method, the following instructions can be used:

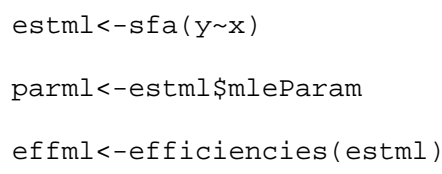

The first line estimates the model with the default options, putting all the results in the object estml. The second line extracts the vector containing the estimations of the parameters from the object estml, which is then saved as the vector parml. Finally, in the last line the individual efficiencies are estimated and saved as effml. It is important to point out that the function efficiencies, since it is used with the default options, estimates $\exp \left(-u_{i}\right)$, following the formula in Jondrow et al. (1982).

\section{B) Bayesian estimation.}

The text file, which we have called SFA_HalfNormal.txt and saved in directory C: , and which includes the code necessary for the estimation of the Half-Normal stochastic frontier production model with WinBUGS, contains the following:

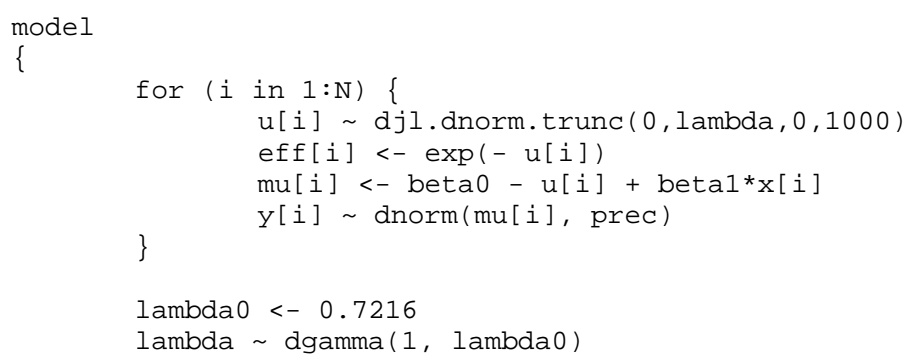




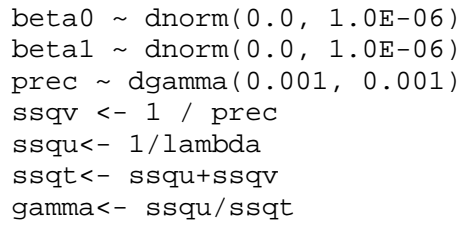

The vector containing the term of inefficiencies is $u$; in order to define a Half-Normal distribution, a particular case of the truncated normal distribution through the command dj1.dnorm.trunc $(0,1$ ambda, 0,1000$)$ is used. It is important to observe that this distribution is not in WinBUGS by default; to be able to use it, an additional component from the web site http://www.winbugs-development.org.uk/shared.html must be downloaded. In addition to the parameters and vectors needed for the definition of the model, the vector eff is also considered, which contains the efficiencies, and the scalars ssqt and gamma for the parameters $\sigma^{2}$ and $\gamma$ respectively.

The R code employed to carry out the Bayesian estimation process is:

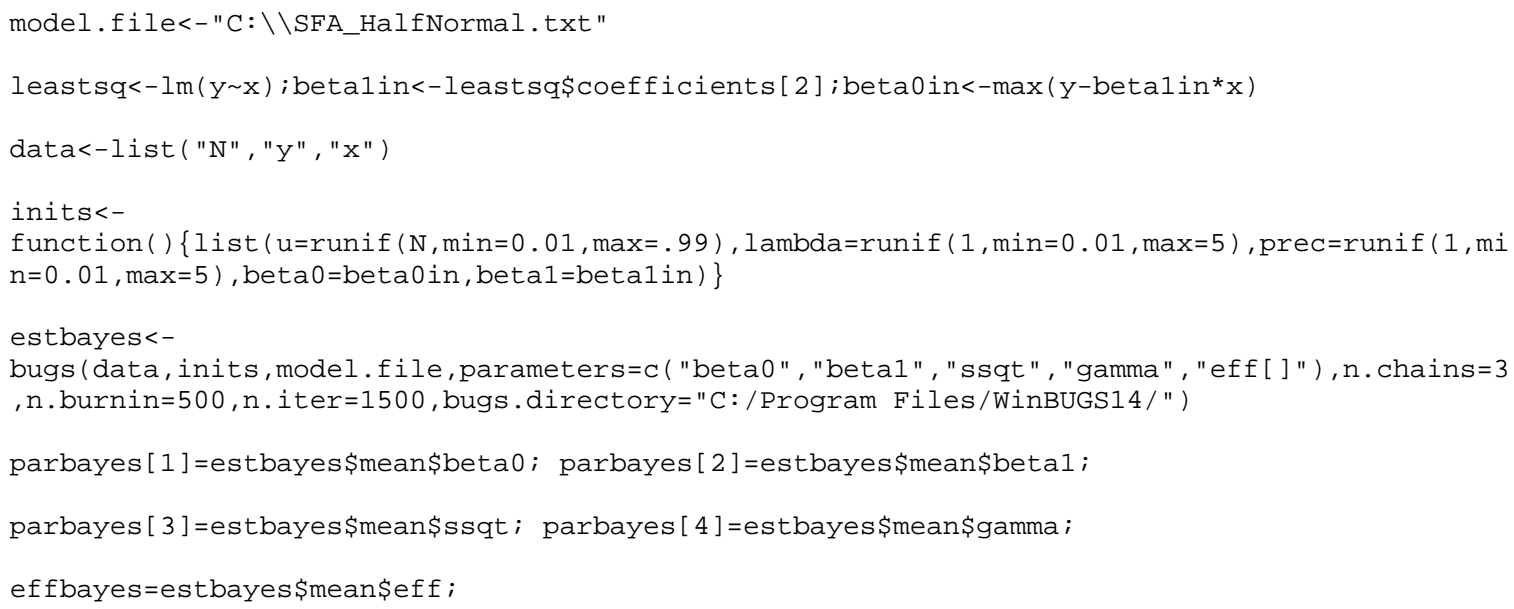

In the first instruction, the text file with the instructions for WinBUGS is loaded; in the second, initial values for $\beta_{0}$ and $\beta_{1}$ are obtained; in the third instruction, the list of data is generated ( $\mathrm{N}$ is the sample size); in the fourth, initial values for the Markov chains are created. In the fifth instruction, the program WinBUGS is called, and data and options are transferred. Let us point out that the option parameters contains the list of parameters of 
interest and the option bugs.directory indicates the directory where the program WinBUGS is installed. The result of the estimation is saved in the object estbayes. In instructions 6 and 7, the vector parbayes is created, which contains the estimations of the parameters. Finally, in instruction 8, the estimated efficiencies are saved in the vector effbayes.

\section{APPENDIX 2}

In this appendix, the complete results of the Monte Carlo experiment are presented.

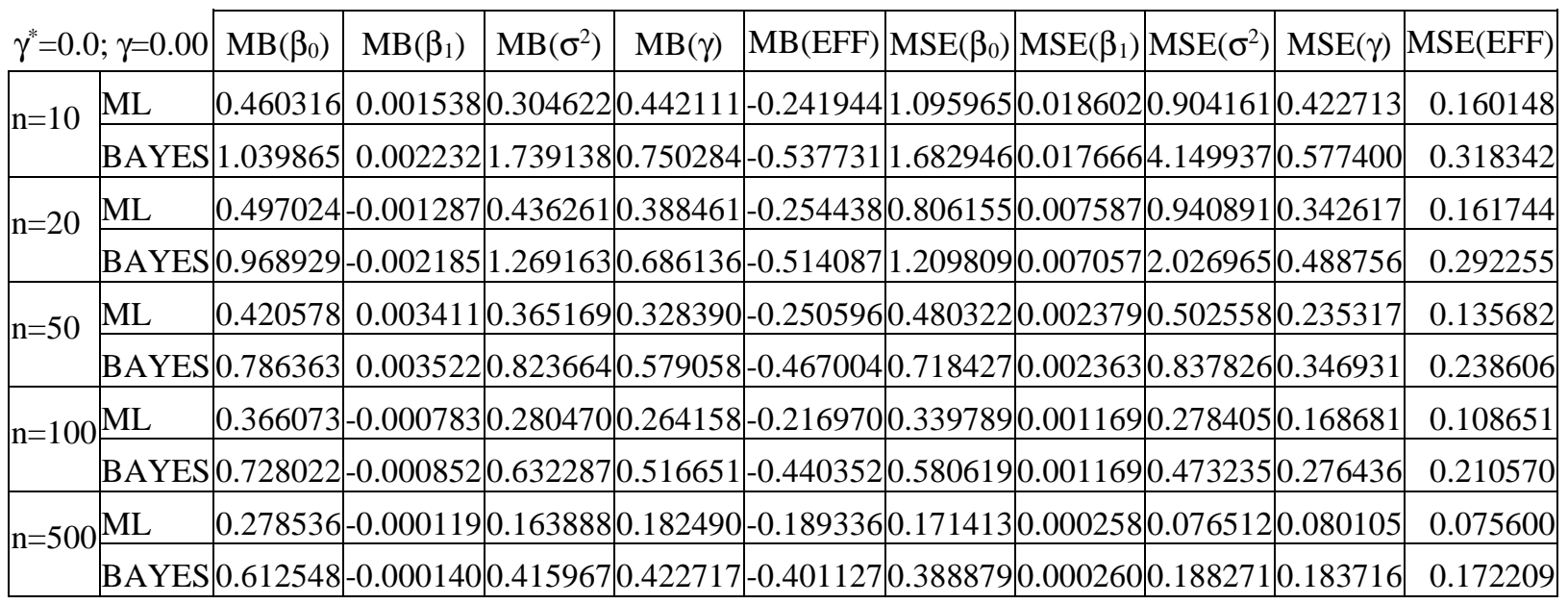

\begin{tabular}{|c|c|c|c|c|c|c|c|c|c|}
\hline \multicolumn{2}{|c|}{$\gamma^{*}=0.1 ; \gamma=0.24$} & $\operatorname{MB}\left(\beta_{0}\right)$ & $\mathrm{B}\left(\beta_{1}\right)$ & $\operatorname{IB}\left(\sigma^{2}\right)$ & $\mathrm{B}(\gamma)$ & $\mathrm{B}(\mathrm{EFF}) \mathrm{M}$ & $\operatorname{MSE}\left(\beta_{1}\right) \operatorname{MSE}\left(\sigma^{2}\right)$ & $\operatorname{MSE}(\gamma)$ & F) \\
\hline \multirow{2}{*}{$n=10$} & ML & 0.033027 & 0.002959 & 103 & 0.190801 & 0.067 & 100 & 034 & 0.122353 \\
\hline & BAYES & 0.622824 & 0.001070 & 1.4 & 0.535366 & 20.8 & 0.0 & 52 & 102940 \\
\hline \multirow{2}{*}{$\mathrm{n}=20$} & ML & 0.048254 & 4 & & 0.140366 & 20. & 10 & 663 & 0.117180 \\
\hline & BAYES & 0. & 0.0 & & & 860.5 & 1 & 96 & 044 \\
\hline \multirow[t]{2}{*}{$=50$} & ML & & & & 106435 & 0.05 & & 86 & \\
\hline & BAYES & 0.391646 & -0.4 & 0.60 & 0.3 & -0.1644600 .2 & 0.0 & 205 & 7517 \\
\hline \multirow[t]{2}{*}{$\mathrm{n}=100$} & ML & -0.005603 & 0.0 & 0.11 & 0.070146 & 0.0556660 .173 & 0.00 & 672 & 0.086151 \\
\hline & BAYES & 0.3 & 0.0011 & 0. & 6 & -0.1377160 .12 & 0.0 & 7808 & 0.055879 \\
\hline \multirow[t]{2}{*}{$\mathrm{n}=500$} & ML & -0.0637 & 221 & 0.02 & -0.000495 & 0.0710730 .087876 & 0.0002250 .0 & 449 & 0.069970 \\
\hline & BAYES & 0.205772 & -0.000222 & 0.234200 & 0.219245 & -0.0985460 .054220 & 0.0002240 .067131 & 0.053252 & 0.043221 \\
\hline
\end{tabular}

\begin{tabular}{|l|l|l|l|l|l|l|l|l|l|l|}
$\gamma^{*}=0.2 ; \gamma=0.41$ & $\operatorname{MB}\left(\beta_{0}\right)$ & $\operatorname{MB}\left(\beta_{1}\right)$ & $\operatorname{MB}\left(\sigma^{2}\right)$ & $\operatorname{MB}(\gamma)$ & $\operatorname{MB}(\operatorname{EFF})$ & $\operatorname{MSE}\left(\beta_{0}\right)$ & $\operatorname{MSE}\left(\beta_{1}\right)$ & $\operatorname{MSE}\left(\sigma^{2}\right)$ & $\operatorname{MSE}(\gamma)$ & $\operatorname{MSE}(\operatorname{EFF})$ \\
\hline
\end{tabular} 


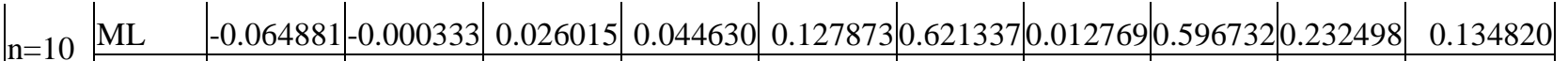
\begin{tabular}{|l|l|l|l|l|l|l|l|l|l|l|l|}
\hline BAYES & 0.473357 & 0.000858 & 1.210748 & 0.384517 & -0.160607 & 0.619603 & 0.011936 & 2.152052 & 0.158025 & 0.074946 \\
\hline
\end{tabular}

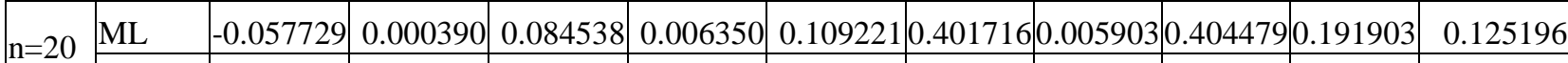
\begin{tabular}{|l|l|l|l|l|l|l|l|l|l|l|l|l|l|}
\hline BAYES & 0.391411 & -0.000732 & 0.822208 & 0.329797 & -0.137886 & 0.346992 & 0.005690 & 0.931648 & 0.122358 & 0.065018 \\
\hline
\end{tabular} \begin{tabular}{|l|l|l|l|l|l|l|l|l|l|l|l|l|l|}
\hline $\mathrm{n}=50$ & $\mathrm{ML}$ & -0.073231 & 0.001305 & 0.087553 & -0.019995 & 0.095381 & 0.249557 & 0.002138 & 0.251139 & 0.144965 & 0.107439 \\
\cline { 2 - 7 } & &
\end{tabular} \begin{tabular}{|l|l|l|l|l|l|l|l|l|l|l|l|l|l|}
\hline BAYES & 0.248183 & 0.001141 & 0.465756 & 0.237435 & -0.098188 & 0.144575 & 0.002113 & 0.316484 & 0.069506 & 0.052311 \\
\hline
\end{tabular}

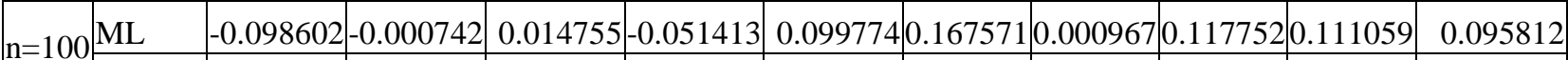
\begin{tabular}{|l|l|l|l|l|l|l|l|l|l|l|l|l|l|} 
BAYES & $0.177449-0.000884$ & 0.286611 & 0.179436 & -0.070002 & 0.072614 & 0.000970 & 0.125296 & 0.042206 & 0.044574 \\
\hline
\end{tabular}

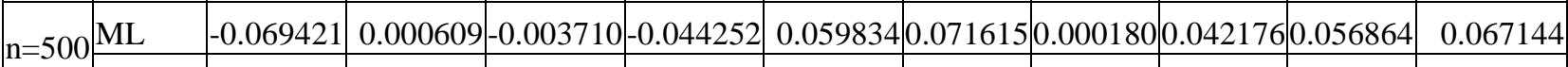
\begin{tabular}{|l|l|l|l|l|l|l|l|l|l|l|l|l|l|l|}
\hline BAYES & 0.093729 & 0.000618 & 0.135371 & 0.103056 & -0.039638 & 0.019800 & 0.000180 & 0.032455 & 0.017515 & 0.039265 \\
\hline
\end{tabular}

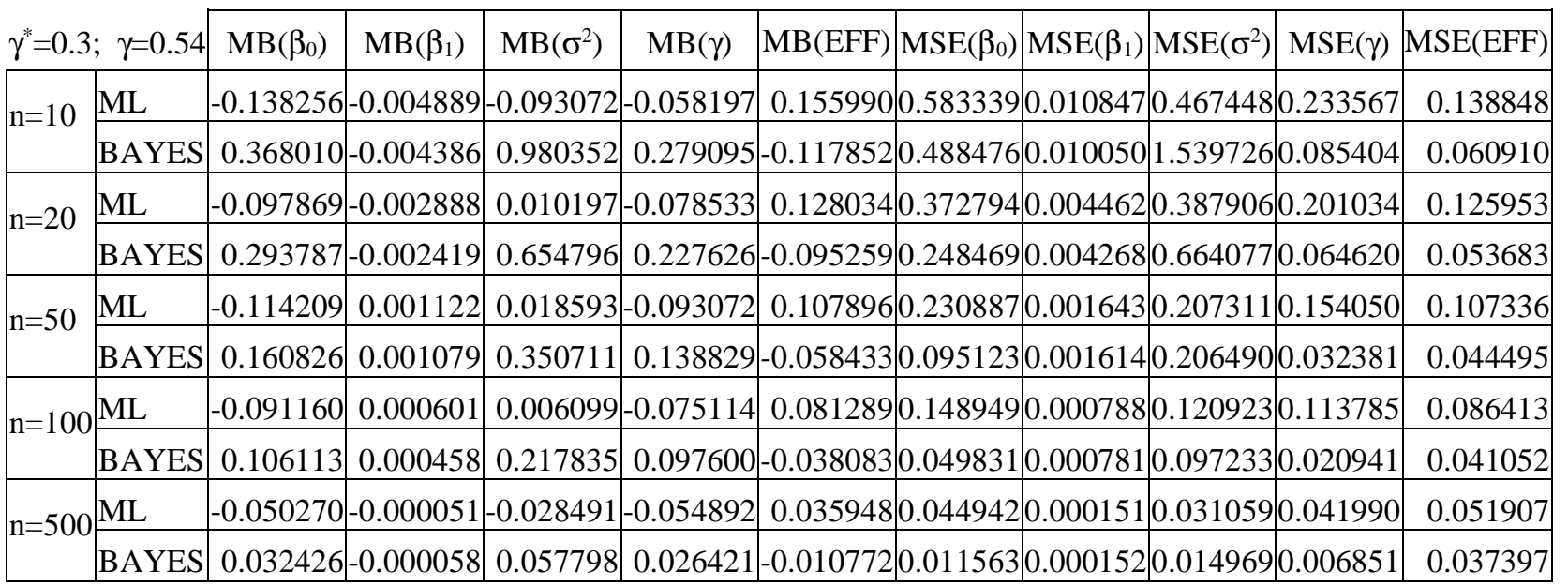

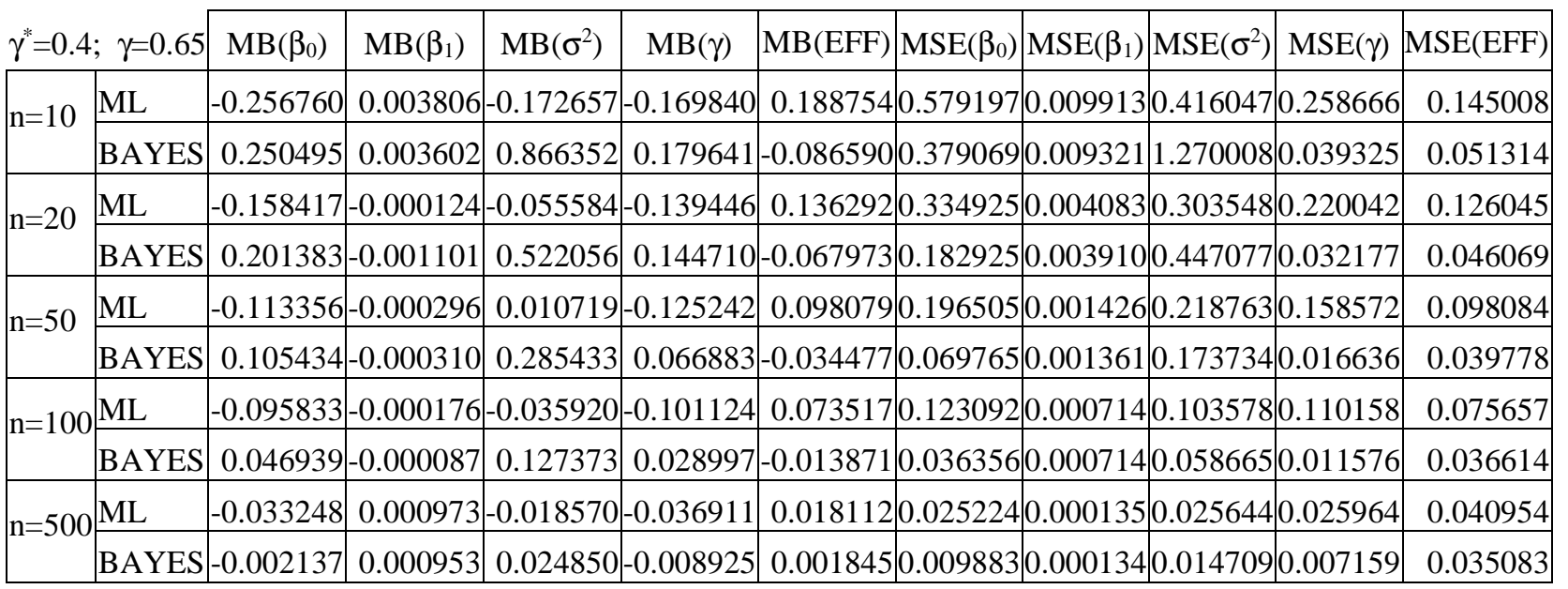

\begin{tabular}{|c|c|c|c|c|c|c|c|c|c|c|}
$\gamma^{*}=0.5 ; \gamma=0.73$ & $\operatorname{MB}\left(\beta_{0}\right)$ & $\operatorname{MB}\left(\beta_{1}\right)$ & $\operatorname{MB}\left(\sigma^{2}\right)$ & $\operatorname{MB}(\gamma)$ & $\operatorname{MB}(\operatorname{EFF})$ & $\operatorname{MSE}\left(\beta_{0}\right)$ & $\operatorname{MSE}\left(\beta_{1}\right)$ & $\operatorname{MSE}\left(\sigma^{2}\right)$ & $\operatorname{MSE}(\gamma)$ & $\operatorname{MSE}(\operatorname{EFF})$ \\
\hline
\end{tabular} \begin{tabular}{|l|l|l|l|l|l|l|l|l|l|l|l|l|l|}
\hline $\mathrm{n}=10$ & ML & -0.232003 & -0.001063 & -0.193776 & -0.178594 & 0.174795 & 0.485236 & 0.008914 & 0.373728 & 0.258971 & 0.133548 \\
\cline { 2 - 7 } &
\end{tabular}

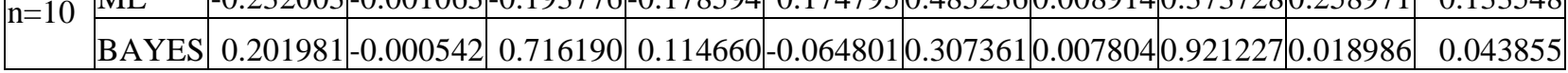


\begin{tabular}{|l|l|l|l|l|l|l|l|l|l|l|l|}
$\mathrm{n}=20$ & $\mathrm{ML}$ & -0.178417 & 0.002649 & -0.091816 & -0.172730 & 0.133880 & 0.324076 & 0.004084 & 0.298974 & 0.226045 & 0.119118 \\
\cline { 2 - 6 } &
\end{tabular} \begin{tabular}{|l|l|l|l|l|l|l|l|l|l|l|l|}
\hline BAYES & 0.148460 & 0.001535 & 0.440077 & 0.088660 & -0.051018 & 0.154261 & 0.003731 & 0.369117 & 0.017827 & 0.040914 \\
\hline
\end{tabular}

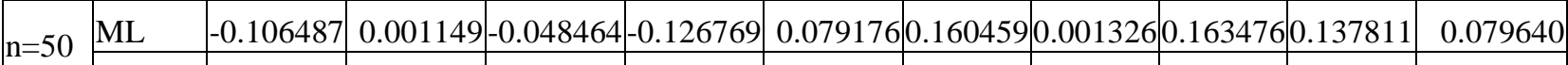
\begin{tabular}{|l|l|l|l|l|l|l|l|l|l|l|l|l|} 
BAYES & 0.063486 & 0.000611 & 0.187754 & 0.023129 & -0.018980 & 0.058683 & 0.001262 & 0.106925 & 0.011788 & 0.035236 \\
\hline
\end{tabular}

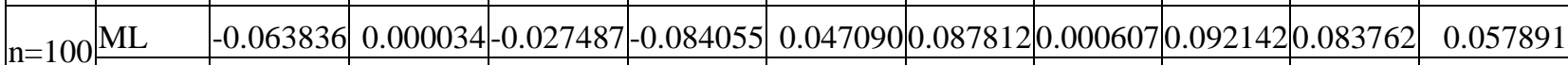
\begin{tabular}{|l|l|l|l|l|l|l|l|l|l|l|l|l|}
\hline BAYES & 0.023777 & 0.000027 & 0.093461 & -0.007140 & -0.004531 & 0.029751 & 0.000602 & 0.053079 & 0.009982 & 0.033408 \\
\hline
\end{tabular}

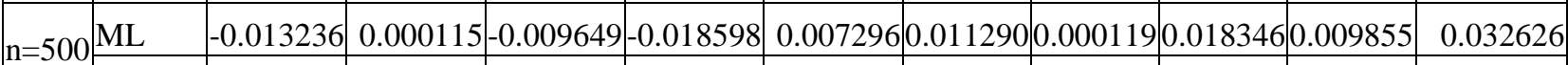
\begin{tabular}{|l|l|l|l|l|l|l|l|l|l|l|l|l|l|}
\hline BAYES -0.008112 & 0.000103 & 0.009164 & -0.020269 & 0.005402 & 0.008310 & 0.000119 & 0.014129 & 0.005843 & 0.031680 \\
\hline
\end{tabular}

\begin{tabular}{c|c|c|c|c|c|c|c|c|c|c|}
$\gamma^{*}=0.6 ; \gamma=0.80$ & $\operatorname{MB}\left(\beta_{0}\right)$ & $\operatorname{MB}\left(\beta_{1}\right)$ & $\operatorname{MB}\left(\sigma^{2}\right)$ & $\operatorname{MB}(\gamma)$ & $\operatorname{MB}(\operatorname{EFF})$ & $\operatorname{MSE}\left(\beta_{0}\right)$ & $\operatorname{MSE}\left(\beta_{1}\right)$ & $\operatorname{MSE}\left(\sigma^{2}\right)$ & $\operatorname{MSE}(\gamma)$ & $\operatorname{MSE}(\operatorname{EFF})$ \\
\hline
\end{tabular}

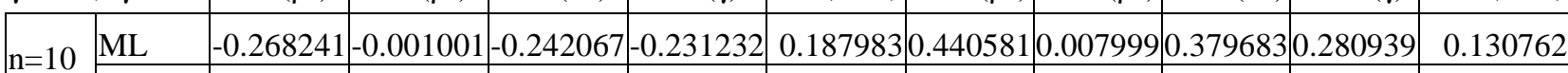

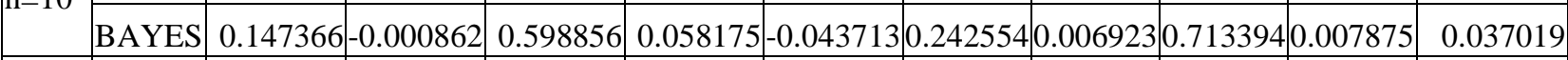
\begin{tabular}{|l|l|l|l|l|l|l|l|l|l|l|l|l|l|}
\hline $\mathrm{n}=20$ & ML & -0.163962 & -0.000557 & -0.121026 & -0.189657 & 0.125883 & 0.298516 & 0.003955 & 0.274649 & 0.218975 & 0.104603 \\
\cline { 2 - 7 }
\end{tabular} \begin{tabular}{|l|l|l|l|l|l|l|l|l|l|l|l|l|l|}
$\mathrm{n}=20$ & BAYES & 0.112294 & -0.000615 & 0.356862 & 0.039957 & -0.033493 & 0.138085 & 0.003498 & 0.291989 & 0.009448 & 0.034060 \\
\cline { 2 - 7 }
\end{tabular}

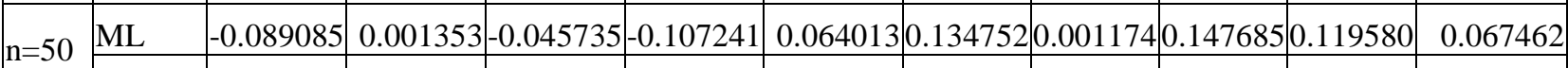

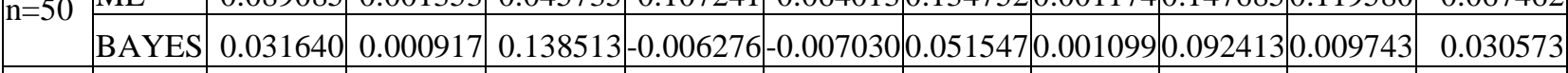

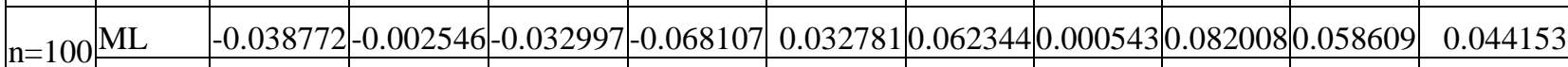
\begin{tabular}{|l|l|l|l|l|l|l|l|l|l|l|l|}
\hline BAYES & 0.010742 & -0.002537 & 0.054066 & -0.029513 & 0.004189 & 0.028077 & 0.000535 & 0.049070 & 0.010155 & 0.029640 \\
\hline
\end{tabular}

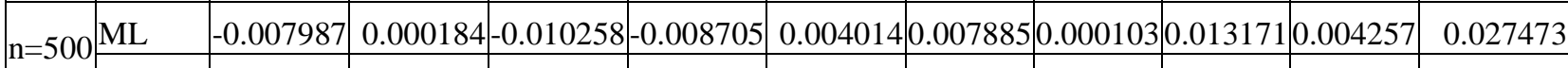
\begin{tabular}{|l|l|l|l|l|l|l|l|l|l|l|l|l|l|l|l|l|l|l|}
\hline BAYES -0.008740 & 0.000198 & 0.000089 & -0.016863 & 0.004650 & 0.007402 & 0.000103 & 0.011777 & 0.003954 & 0.027383 \\
\hline
\end{tabular}

\begin{tabular}{|c|c|c|c|c|c|c|c|c|c|c|c|}
\hline \multicolumn{2}{|c|}{$\gamma^{*}=0.7 ; \gamma=0.87$} & $\mathrm{M}$ & $\operatorname{MB}\left(\beta_{1}\right)$ & 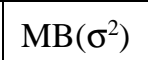 & $\operatorname{MB}(\gamma)$ & F) & $\operatorname{MSE}\left(\beta_{0}\right)$ & $\operatorname{MSE}\left(\beta_{1}\right)$ & $\operatorname{MSE}\left(\sigma^{2}\right)$ & $\operatorname{MSE}(\gamma)$ & LTI \\
\hline \multirow{2}{*}{10} & ML & & & & & & & & 0 & & 0.117906 \\
\hline & BAYES & & & & & & & & & & \\
\hline \multirow[t]{2}{*}{20} & ML & & & & & & & & & & \\
\hline & BAYES & 0.06 & & 0 & 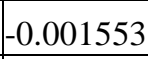 & & & & & & 244 \\
\hline \multirow{2}{*}{$=50$} & ML & -0.0 & 0. & 31 & & & & & & & 5503 \\
\hline & BAYES & 0.0 & 615 & & & & & & & & 5177 \\
\hline \multirow[t]{2}{*}{$\mathrm{n}=10$} & ML & & & & & & & & & & 433 \\
\hline & BA & & 0.00 & & & & & & & & 581 \\
\hline \multirow[t]{2}{*}{500} & ) & -0.0 & 0.0 & & & & & & & & 1122 \\
\hline & & & & & & & & & & & 0.02 \\
\hline
\end{tabular}

\begin{tabular}{|c|c|c|c|c|c|c|c|c|c|}
\hline \multicolumn{2}{|c|}{$\gamma^{*}=0.8 ; \gamma=0.92$} & $\operatorname{MB}\left(\beta_{0}\right)$ & $\operatorname{MB}\left(\beta_{1}\right)$ & $\operatorname{MB}\left(\sigma^{2}\right)$ & $\operatorname{MB}(\gamma)$ & $\operatorname{MB}(\mathrm{EFF}) \operatorname{MSE}\left(\beta_{0}\right)$ & $\operatorname{MSE}\left(\beta_{1}\right) \operatorname{MSE}\left(\sigma^{2}\right)$ & $\operatorname{MSE}(\gamma)$ & MSE(EFF) \\
\hline \multirow{2}{*}{$n=10$} & ML & -0.332012 & 0.003221 & -0.300147 & -0.263047 & 0.1878710 .432689 & 0.0066560 .361155 & 0.273858 & 0.113713 \\
\hline & BAYES & 0.012059 & 0.002277 & 0.407870 & -0.037582 & -0.0046960 .1 & 220.44 & 0.004270 & 0.025304 \\
\hline \multirow[t]{2}{*}{$n=20$} & ML & -0.155127 & 0.000148 & -0.139493 & -0.150360 & \begin{tabular}{|l|l|}
0.093837 & 0.18668 \\
\end{tabular} & 0.0025070 .224959 & 0.154430 & 0.067722 \\
\hline & BAYES & 0.010898 & -0.000151 & 0.204089 & -0.030152 & $|-0.001861| 0.071123$ & 0.0020250 .175164 & 0.005399 & 0.020870 \\
\hline
\end{tabular}




\begin{tabular}{|l|l|l|l|l|l|l|l|l|l|l|l|l|}
$\mathrm{n}=50$ & ML & -0.037513 & -0.000391 & -0.036609 & -0.052147 & 0.026765 & 0.065707 & 0.000873 & 0.102198 & 0.048751 & 0.032163 \\
\cline { 2 - 10 } & BAYES & -0.001754 & -0.000308 & 0.063953 & -0.036266 & 0.004917 & 0.034229 & 0.000791 & 0.065456 & 0.007193 & 0.019044 \\
\hline \multirow{2}{*}{$\mathrm{n}=100$} & ML & -0.019291 & 0.000491 & -0.013429 & -0.016434 & 0.008941 & 0.025392 & 0.000410 & 0.048776 & 0.010324 & 0.019448 \\
\cline { 2 - 10 } & BAYES & -0.013366 & 0.000263 & 0.026105 & -0.029760 & 0.006102 & 0.020257 & 0.000403 & 0.038798 & 0.005126 & 0.017610 \\
\hline \multirow{2}{*}{$\mathrm{n}=500$} & ML & -0.001571 & 0.000356 & 0.001291 & -0.000112 & 0.000437 & 0.003888 & 0.000073 & 0.009096 & 0.000601 & 0.015290 \\
\cline { 2 - 9 } & BAYES & -0.002357 & 0.000348 & 0.007200 & -0.005157 & 0.000697 & 0.003927 & 0.000074 & 0.009055 & 0.000683 & 0.015314 \\
\hline
\end{tabular}

\begin{tabular}{|c|c|c|c|c|c|c|c|c|c|c|}
\hline \multicolumn{2}{|c|}{$\gamma^{*}=0.9 ; \gamma=0.96$} & & & & ） & F) 1 & & $2)$ & MS & $\Lambda$ \\
\hline \multirow[t]{2}{*}{10} & & & & & & 7690 & & & & 2091 \\
\hline & BA) & & 6 & & & $74 \mid 0$. & & & & \\
\hline \multirow{2}{*}{20} & ML & & & & & & & & & \\
\hline & & & & & & & & & & \\
\hline \multirow[t]{2}{*}{$=50$} & M & & & & & 680. & & & & \\
\hline & BAY & & & & & 640. & & & & \\
\hline \multirow[t]{2}{*}{$n=100$} & ML & -0.6 & & & & & & & & \\
\hline & & & & & & & & & & \\
\hline \multirow{2}{*}{$n=500$} & ML & -0.0 & 0.0 & & & & & & & 144 \\
\hline & $\mathrm{BA}$ & -0.002875 & 0 & -1 & 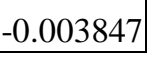 & $345 \mid 0.0$ & & & & 16 \\
\hline
\end{tabular}

\begin{tabular}{|c|c|c|c|c|c|c|c|c|c|c|}
\hline \multicolumn{2}{|c|}{$\gamma^{*}=1.0 ; \gamma=1.00$} & 0) & 1) & $\mathrm{MB}\left(\sigma^{2}\right)$ & $\gamma)$ & $\mathrm{M}$ & ) & $\operatorname{MSE}\left(\sigma^{2}\right)$ & $\operatorname{ISE}(\gamma)$ & \\
\hline & & 90 & 00 & - & 595 & 0.1809020. & 0.0 & & & \\
\hline & AYES & & & & & 1200. & & & & \\
\hline \multirow{2}{*}{$=20$} & & & & & & & & & & \\
\hline & & & & & & & & & & \\
\hline \multirow{2}{*}{50} & & & & & & 440. & & & & \\
\hline & BA & & & & & 880 & & & & \\
\hline \multirow{2}{*}{$=100$} & & 880 & & & & & & & & \\
\hline & & & & & & & & & & \\
\hline \multirow[t]{2}{*}{$1=500$} & & & & & & & & & & 22 \\
\hline & $\mathrm{B}_{2}$ & $\perp$ & 1 & -1 & 1 & 8510. & & & & 00 \\
\hline
\end{tabular}




\section{REFERENCES}

AIGNER, D.J.; CHU, S.F. (1968), “On estimating the industry production function”, American Economic Review, 58, pp. 826-839.

AIGNER, D.J.; LOVELL, C.A.; SCHMIDT, P. (1977), "Formulation and estimation of stochastic frontier production function models", Journal of Econometrics, 6, pp. 21-37.

BATTESE, G.E.; CORRA, G.S. (1977), "Estimation of a production frontier model: With application to the Pastoral Zone of Eastern Australia”, Australian Journal of Agricultural Economics, 21, pp.169-179.

COELLI, T. (1995), "Estimators and hypothesis test for a stochastic frontier function: A Monte Carlo analysis", The Journal of Productivity Analysis, 6, pp. 247-268.

COELLI, T. (1996), A guide to FRONTIER version 4.1: a computer program for frontier production function estimation. CEPA Working Paper 96/07, Department of Econometrics, University of New England, Armidale, Australia. http://www.uq.edu.au/economics/cepa/software/FRONT41-xp1.zip

FARRELL, M.J. (1957), “The measurement of productive efficiency", Journal of the Royal Statistical Society (A), 120, pp. 253-281.

DORFMAN, J.H.; KOOP, G. (2005), "Current developments in productivity and efficiency measurement”, Special issue of Journal of Econometrics, 126, pp.233-570.

GELFAND, A.E.; SMITH, A.F. (1990): "Sampling-based approaches to calculating marginal densities", Journal of the American Statistical Association, 85, pp. 398-409

GREEN, W.H. (1980): "Maximun Likelihood Estimations of econometric frontier functions, Journal of Econometrics, 13, pp. 27-56.

GRIFFIN, J.E.; STEEL, M.F.J. (2007), "Bayesian stochastic frontier analysis using WinBUGS”, Journal of Productivity Analysis, 27, pp. 163-176.

JONDROW, J.; NOVELL, C.A.K.; MATEROV, I.S.; SCHMIDT, P. (1982): “On estimation of technical inefficiency in the stochastic frontier production function model", Journal of Econometrics, 19, pp. 233-238

KOOP, G.; STEEL, F.J. (2003), Koop, G.; Steel, M.F. (2003), "Bayesian analysis of stochastic frontier models". En: Baltagi, B.H. (editor), A companion to theoretical econometrics, Blackwell.

KOOP, G.; STEEL, M.F.J.; OSIEWALSKI, J. (1995), "Posterior analysis of stochastic frontier models using Gibbs sampling”, Computational Statistics, 10, pp. 353-373.

MEEUSEN, W.; VAN DEN BROECK, J. (1977), "Efficiency estimation from Cobb-Douglas production functions with composed error", International Economic Review, 18, pp. 435-444.

ORTEGA, F.J.; BASUlTO, J. (2009), "Estimación Bayesiana en modelos de producción con frontera determinista", Estudios de Economía Aplicada, $27 \quad$ (2), p. 573. http://www.revistaeea.net/documentos/27205.pdf

ORTEGA, F.J.; BASULTO, J.; CAMÚÑEZ, J.A. (2009), “Comparing Bayesian and corrected least-squares estimators in frontier production models", Boletín de Estadística e Investigación Operativa, 25 (2), pp. 8696.

OSIEWALSKI, J.; STEEL, M.F.J. (1998), "Numerical tolos for the Bayesian analysis of stochastic frontier models”, Journal of Productivity Analysis, 10, pp. 133-117.

STURZ, S.; LIGGES, U.; GELMAN, A. (2005), "R2WinBUGS: A package for running WinBUGS from R”, Journal os Statistical Software, 12 (3), pp. 1-16.

VAN DEN BROEK, J.; KOOP, G., OSIEWALSKI, J.; STEEL, M.F.J., (1994), "Stochastic frontier models. A Bayesian perspective", Journal of Econometrics, 61, pp. 273-303. 
ZHANG, X. (1999), “A Monte Carlo study on the finite sample properties of the Gibbs sampling method for a stochastic frontier model”, Journal of Productivity Analysis, 14, pp. 71-83. 